\title{
Ab-initio molecular dynamics study of lanthanides in liquid sodium
}

\author{
Xiang Li (a), Adib Samin (a), Jinsuo Zhang(a)* , C. Unal(b), R. D. Mariani(c)
}

(a) Nuclear Engineering Program, Department of Mechanical and Aerospace Engineering, Ohio State University 201 W 19th Avenue, Columbus, Ohio 43210 USA

(b) Los Alamos National Laboratory, P.O. Box 1663, Los Alamos, NM 87545

(c) Idaho National Laboratory, Materials and Fuels Complex, Idaho Falls, ID 83415, USA

\begin{abstract}
:
To mitigate the fuel cladding chemical interaction (FCCI) phenomena in liquid sodium cooled fast reactors, a fundamental understanding of the lanthanide ( $\mathrm{Ln})$ transport through liquid Na-Cs filled pores in U-Zr fuel is necessary. In this study, we investigate three abundant Ln fission products diffusion coefficients in liquid $\mathrm{Na}$ at multiple temperatures. By utilization of Ab-initio Molecular Dynamics, the Ln diffusivities are found to be in the magnitude order of liquid diffusion $\left(10^{-5} \mathrm{~cm}^{2} / \mathrm{s}\right)$ and the temperature dependence of the diffusivity for different lanthanides in liquid sodium was explored. It is also observed that dilute concentration of Pr and $\mathrm{Nd}$ led to a significant change in $\mathrm{Na}$ diffusivity. The structural and electronic properties of Na-Ln metallic systems have been investigated. The total coordination number shows dependence on both the temperature and the composition.
\end{abstract}

Keywords: Ab-initio molecular dynamics, atomic structure, electronic density of states, diffusion coefficient.

\section{Introduction:}

Metallic fuels have shown to be a prospective candidate for next generation fuels due to their relative ease of production and reprocessing, high burnup and inherent recyclability [1]. One of

\footnotetext{
*Corresponding author: J. Zhang, email: zhang.3558@osu.edu
} 
the main concerns with using such fuels is the clad failure due to Fuel Cladding Chemical Interaction (FCCI). As the fuel swells during burnup, the lanthanide (Ln) fission products with particularly abundant cumulative yield can migrate to the fuel periphery and form low-melting point intermetallic compounds with cladding constituents such as Fe and Ni [2]. This chemical interaction between the fission products and the cladding can not only reduce cladding thickness, but also degrade its load-bearing capability [3]. The FCCI phenomenon is deemed as one of the limiting factors of operation of this particular fuel. Therefore, systematic investigations of the $\mathrm{Ln}$ redistribution and migration to the fuel surface are essential to understand and mitigate FCCI for optimizing the utilization of metallic fuels.

A "liquid-like" transport mechanism for Ln fission products was recently proposed based on the post irradiation experiment (PIE) from Experimental Breeder Reactor-II (EBR-II) [4]. This model is based on the idea that fission products may transport as solutes in liquid metals such as liquid cesium or cesium-sodium mixture. The mechanism can qualitatively account for the Ln migration to the fuel slug peripheral surface and their deposition with a sludge-like form [4]. To better describe the Ln transport behavior and examine the liquid-like mechanism, the acquisition of fundamental data such as Ln diffusion coefficients in liquid sodium is necessary. Moreover the knowledge of diffusivity in liquid metals is important for prediction of mass transfer rates which plays an important role in several applications such as corrosion [5], phase separation [6], and crystal growth [7]. However, the currently available data from experimental measurements is limited due to the difficulties associated with high temperature experiments and challenge of maintaining the integrity of the samples during the transfer of liquid metals between apparatuses [8]. Therefore, simulation methods provide useful and powerful alternatives to calculate the thermodynamic and transport properties for these systems at elevated temperatures. 
In this study the Ab-initio Molecular Dynamics (AIMD) technique is utilized to study the diffusivity of three Ln elements in liquid sodium at multiple characteristic temperatures during reactor operation. In addition, we investigate the effects of Ln impurity atoms on the properties of liquid sodium relevant to their application in nuclear reactors.

\section{Computational Details:}

The AIMD simulations were performed by using the Vienna ab initio simulation package (VASP) $[9,10]$. The projector augmented wave (PAW) method was employed within the density functional theory framework and Perdew-Burke-Ernzerhof (PBE) generalized gradient approximation (GGA) was used for the exchange-correlation functional [11]. The first order Methfessel-Paxton's smearing method was used with a width of $0.1 \mathrm{eV}$ to determine the partial occupancies for each wavefunction [12]. The cutoff energy of $400 \mathrm{eV}$ was applied and convergence of energy $10^{-6} \mathrm{eV}$ was set. Spin polarization effects were not considered in this calculation. The Brillouin zone was sampled through the gamma point which is acceptable for large and disordered systems in this case. The AIMD simulations were performed with a time step of $2 \mathrm{fs}$ in the canonical ensemble (NVT) and the Nose-Hoover thermostat was applied with a relaxation time of $20 \mathrm{fs}$. The systems were initially equilibrated for $5 \mathrm{ps}$ before any statistics of interest were collected for further calculations. The molecular dynamic model of liquid metal was constructed with 128 atoms in the supercell. For binary alloy systems, 4 Ln atoms were introduced in replacement of $\mathrm{Na}$ atoms since we only focus on the case with low $\mathrm{Ln}$ concentration in the reactor application. Moreover, variation of low Ln concentrations was proved to have negligible effects on the structural or electronic properties in our previous study [13]. 


\section{Results and Discussion:}

\subsection{Atomic structure}

The structure of the liquid metal is characterized by the form of its pair correlation function (PCF). Fig. 1 shows the calculated total pair correlation functions $g(r)$ of different Na-based metallic systems, which can be obtained by [14]:

$$
\begin{aligned}
g(\mathrm{r}) \approx & c_{N a N a} g_{N a N a}(r)+c_{N a L n} g_{N a L n}(r)+c_{L n N a} g_{L n N a}(r) \\
& +c_{L n L n} g_{L n L n}(r)
\end{aligned}
$$

where $g_{i j}(r)$ are four partial pair distribution functions of Na-Na, Na-Ln, Ln-Na, Ln-Ln pairs. The weighting factors can be expressed as $x_{i} x_{j} Z_{i} Z_{j} /\left(x_{N a} Z_{N a}+x_{L n} Z_{L n}\right)^{2}$ with the atomic number of an atom $i, Z_{i}$ and the corresponding concentration $x_{i}$. In Fig. 1 no well-defined first coordination shell is observed in the $g(r)$ for pure liquid $\mathrm{Na}$, which reflects the deviation from a crystal structure. The liquid binary alloys, Na-Pr and Na-Nd, present a similar shape of PCF as liquid $\mathrm{Na}$ and therefore show approximately homogeneous metallic properties. In the $\mathrm{Na}-\mathrm{Ce}$ binary system, however, segregation phenomena have been observed with the appearance of an extra peak at a distance of around $2.65 \AA ̊$ at $723 \mathrm{~K}$. As shown in Fig. 2 , this peak was found to mainly come from the contribution of the partial pair correlation function, $g_{\text {Cece }}(r)$ with a peak indicating aggregation of the $\mathrm{Ce}$ atoms and the $\mathrm{Ce}$ separation from the $\mathrm{Na}$ solvent atoms. The $\mathrm{Na}-\mathrm{Ce}$ atomic structure after 60 ps simulation in Fig. 3 shows that all the four $\mathrm{Ce}$ atoms gather together during diffusion. This observation, known as segregation, is caused by the rejection of the solutes from a solidified alloy into the liquid phase due to different impurity solubility in 
liquid and solid phases at the equilibrium temperature. Segregation can be used to predict the solidification [15] and the degree of segregation depends on the chemical composition of the alloy [16]. Therefore, the Na-Ce alloys at the temperatures considered in this study are expected to be in the amorphous solid state while $\mathrm{Na}-\mathrm{Pr}$ and $\mathrm{Na}-\mathrm{Nd}$ still maintain liquid features. Moreover, with increasing temperature the height of the peaks of $g(r)$ decreases and the peak position shifts to a shorter distance. This observation agrees with the results from a previous study on the simulation of liquid alkali metals [17].

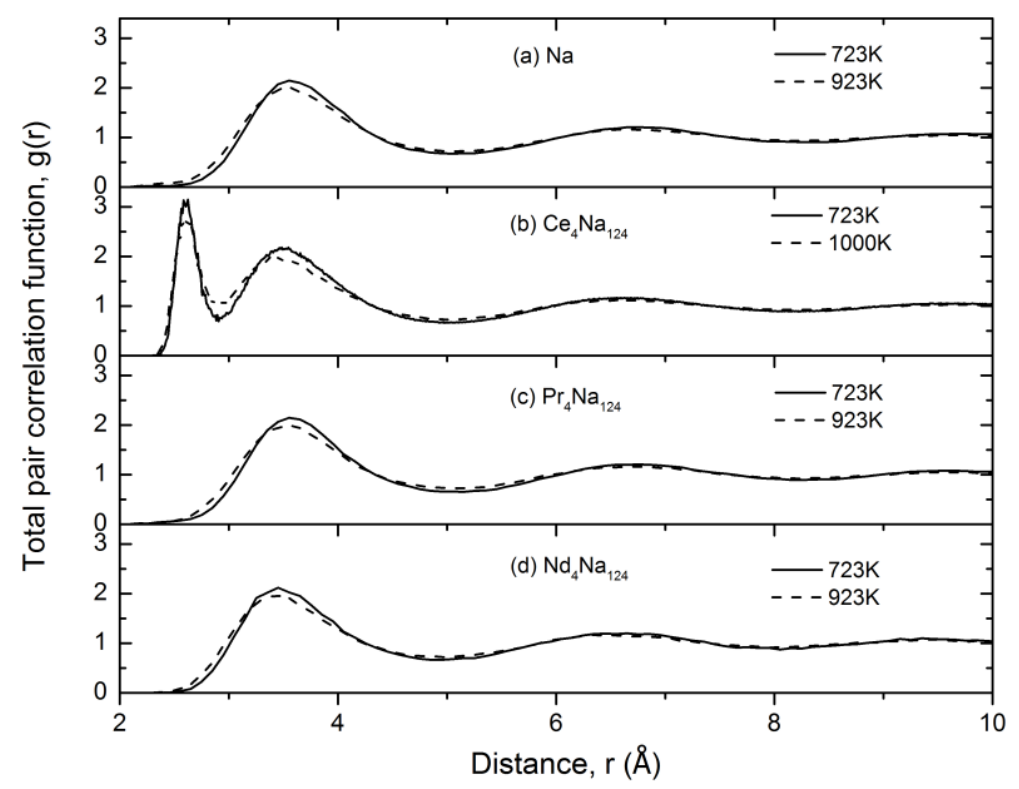

Fig. 1. The total pair correlation functions $\boldsymbol{g}(\boldsymbol{r})$ of four liquid metallic systems. PCF are calculated at two temperatures for each metallic system. 


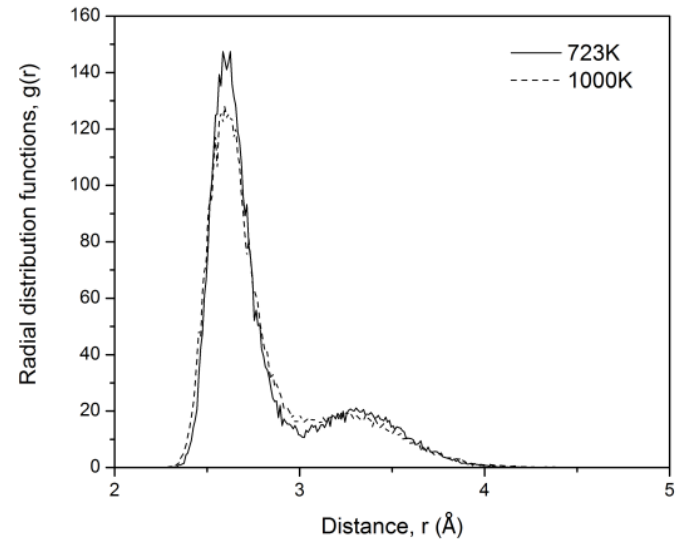

Fig. 3. The Ce-Ce pair correlation functions $g(r)$ at two temperatures.

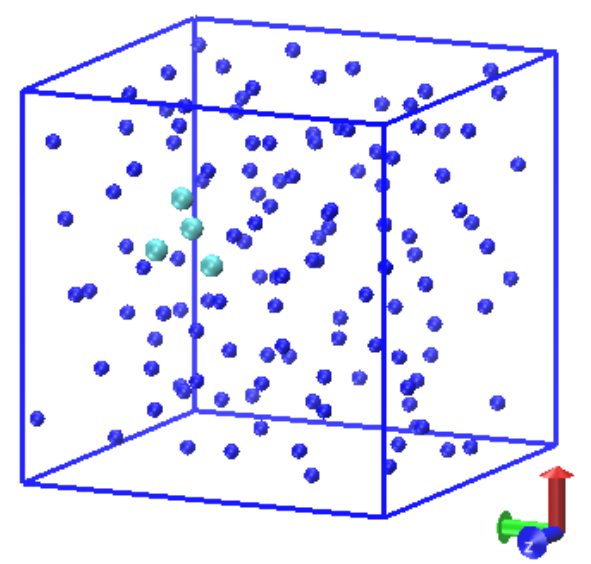

Fig. 2. A snapshot of atomic structure of $\mathrm{Ce}_{4} \mathrm{Na}_{124}$ system after $60 \mathrm{ps}$ at $723 \mathrm{~K}$ with four Ce (cyan atoms) aggregated.

Based on the obtained $g(r)$, we calculated the total coordination number as a weighted sum of the coordination numbers of four atomic pairs with a similar method as equation (1). In Table I, the calculated coordination number of $\mathrm{Na}$ with density $0.9674 \mathrm{~g} / \mathrm{cm}^{3}$ agrees well with the value $13.0 \pm 0.1$ at $573 \mathrm{~K}$ with a density $0.8688 \mathrm{~g} / \mathrm{cm}^{3}$ from a previous molecular dynamic simulation on liquid sodium [18]. We note that both the introduction of Ln atoms and the temperature increase can cause a reduction of the coordination number. Among three Na-Ln alloys, the coordination number increases with the increasing Ln atomic number and this may be caused by the effects of atomic size on the coordination number in amorphous metals [19]. However, since $\mathrm{Na}-\mathrm{Pr}$ and Na-Nd in our current study still show liquid characteristics, more data at lower temperatures where both systems reach the amorphous state is required to investigate the relationship between coordination number and the size of $\mathrm{Ln}$ atom. 
Table I Total coordination number of various Na-based liquid metal systems at different temperatures.

\begin{tabular}{ccc}
\hline System & Temperature (K) & Coordination Number \\
\hline \multirow{2}{*}{$\mathrm{Na}$} & 723 & $13.08 \pm 0.17$ \\
& 923 & $12.89 \pm 0.19$ \\
\hline \multirow{2}{*}{$\mathrm{Ce}$ in $\mathrm{Na}$} & 723 & $10.63 \pm 0.11$ \\
& 1000 & $10.45 \pm 0.11$ \\
\hline \multirow{2}{*}{$\mathrm{Pr}$ in $\mathrm{Na}$} & 723 & $11.02 \pm 0.14$ \\
& 923 & $10.79 \pm 0.13$ \\
\hline \multirow{2}{*}{$\mathrm{Nd}$ in $\mathrm{Na}$} & 723 & $11.18 \pm 0.12$ \\
& 923 & $10.91 \pm 0.13$ \\
\hline
\end{tabular}

\subsection{Electronic density of states}

The addition of Ln atoms has an impact on the electronic properties, which can be observed by examining the electronic density of states (DOS). Fig. 4 shows the electronic DOS of four different liquid metallic configurations after 5ps equilibration and the origin of the energy is taken to be the Fermi level $\left(E_{F}=0\right)$. Local minimum positions can always be found below the Fermi level in each metallic system which reflects a deviation from the model of almost free electrons. Our results for the pure sodium system agree well with the previous Ab-initio MD work [17]. The addition of $\mathrm{Ln}$ atoms results in a more concentrated DOS around the Fermi energy and several distinct peaks have been observed. This is consistent with the former DFT calculation on electronic structures of Ce impurities in different hosts such as $\mathrm{Cu}, \mathrm{Ag}$ and $\mathrm{Pd}$ where DOS curves with similar characteristics were obtained [20]. 


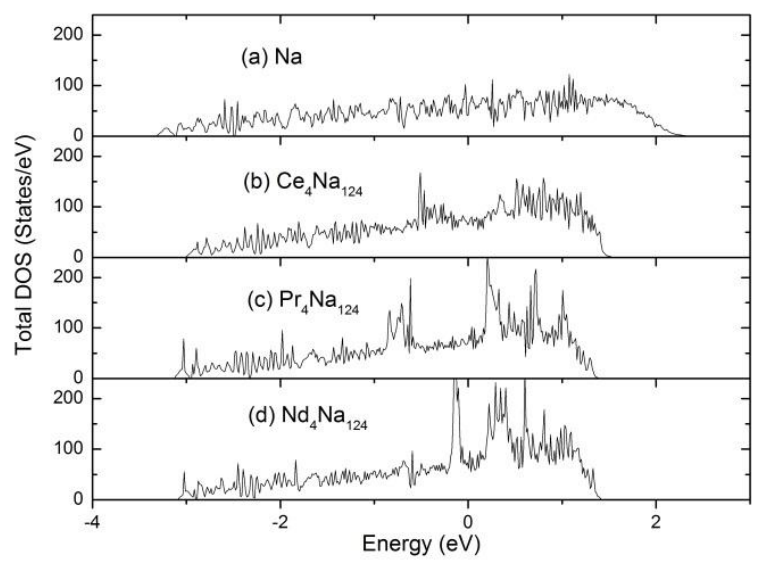

Fig. 4. The electronic density of states (DOS) of four liquid metallic systems at $723 \mathrm{~K}$. The origin of the energy is adjusted to be the Fermi level.

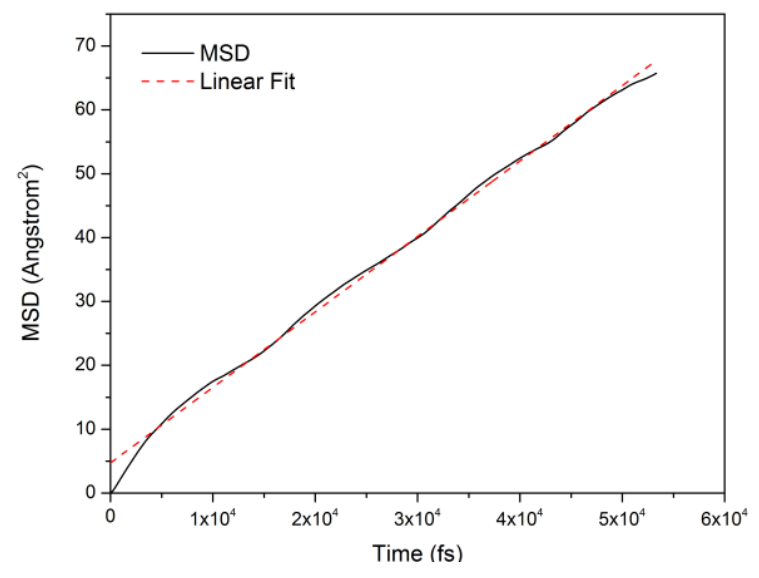

Fig. 5. The example mean squared displacements of $4 \mathrm{Ce}$ atoms in $124 \mathrm{Na}$ atoms at $723 \mathrm{~K}$ are recorded against time in femtoseconds.

\subsection{Diffusion coefficients at different temperatures}

The diffusivity of Ln in liquid metals can be calculated from the Ln mean-squared displacement (MSD) based on the Einstein-Stokes equation [21]:

$$
D=\lim _{\mathrm{t} \rightarrow \infty} \frac{\sigma(\mathrm{t})}{6 \mathrm{t}}
$$

where $\sigma(\mathrm{t})$ is the averaging mean-square displacement in a time period of $\mathrm{t}$. As shown in Fig. 5, the diffusion coefficient for $\mathrm{Ce}$ in liquid $\mathrm{Na}$ at $723 \mathrm{~K}$ was calculated by recording mean square displacements of four $\mathrm{Ce}$ atoms in the liquid metallic system of $124 \mathrm{Na}$ atoms for more than 50 ps. The slope of the fitting data can be used to obtain the diffusivities for three Ln atoms at different temperatures as listed in Table II. The diffusion coefficients of $\mathrm{Ln}$ in liquid Na were all found to be in the liquid diffusion magnitude order of $10^{-5} \mathrm{~cm}^{2} / \mathrm{s}$ which can be applied to validate the "liquid-like" transport mechanism. Also the diffusivity for each metallic system increases with the temperature as expected. The corresponding experimental values are still 
lacking. However, other experimental data for diffusion of specific Ln in other similar systems at elevated temperatures can provide some supports. For example, our simulation data show consistence with the previous experimental studies on the diffusion of Cerium oxy ions in $\mathrm{MgCl}_{2}-\mathrm{NaCl}-\mathrm{KCl}$ eutectic at a temperature of $853 \mathrm{~K}$ which yields a diffusion coeffcient in the magnitude order of $10^{-5} \mathrm{~cm}^{2} / \mathrm{s}$ [22]. Moreover, the diffusivity of $\mathrm{K}$ in the system with composition $\mathrm{Na}_{90} \mathrm{Li}_{10}$ at $373 \mathrm{~K}$ is calculated to be $4.77 \times 10^{-5} \mathrm{~cm}^{2} / \mathrm{s}$ in a classical molecular study of the Na-K alloy [23].

Table II shows that the diffusion coefficient of $\mathrm{Ce}$ has the strongest dependence on the temperature. One possible reason is that Na-Ce might be in the intermediate state between solid and liquid as discussed above and an increase in temperature facilitates transition of the metallic system to liquid state with larger diffusion coefficient. Furthermore, no conclusions may be drawn regarding the correlation between atomic number and diffusivity in liquid sodium. A summary of Na diffusivity in multiple metallic systems is shown in Table III. The self-diffusivity of pure $\mathrm{Na}$ at $723 \mathrm{~K}$ is consistent with the values from previous calculation and experiments considering the errors generated during simulation. Generally, a decrease of Na diffusivity is caused by the inclusion of $\mathrm{Ln}$ atoms. Compared with $\mathrm{Pr}$ and $\mathrm{Nd}$, Ce has a smaller effect on $\mathrm{Na}$ diffusivity. This may be due to the segregation phenomenon in $\mathrm{Na}-\mathrm{Ce}$ system and $\mathrm{Na}$ atoms are less influenced by the concentrated Ce atoms.

Table II Ln diffusivity in liquid Na metallic system at different temperatures.

\begin{tabular}{ccc}
\hline System & Temperature (K) & Diffusivity $\left(\mathrm{cm}^{2} / \mathrm{s}\right) \times 10^{5}$ \\
\hline \multirow{2}{*}{$\mathrm{Ce}$ in $\mathrm{Na}$} & 523 & $1.04 \pm 0.07$ \\
& 723 & $1.97 \pm 0.14$ \\
& 1000 & $5.34 \pm 0.13[13]$ \\
\hline \multirow{2}{*}{ Pr in Na } & 723 & $4.53 \pm 0.29$ \\
& 923 & $6.01 \pm 0.34$ \\
\hline \multirow{2}{*}{$\mathrm{Nd}$ in Na } & 723 & $4.00 \pm 0.27$ \\
& 923 & $4.63 \pm 0.28$ \\
\hline
\end{tabular}


Table III Na diffusivity in various metallic systems at different temperatures

\begin{tabular}{ccc}
\hline System & Temperature $(\mathrm{K})$ & Diffusivity $\left(\mathrm{cm}^{2} / \mathrm{s}\right) \times 10^{5}$ \\
\hline \multirow{2}{*}{$\mathrm{Na}$} & 723 & $12.76 \pm 0.51$ \\
& 923 & $17.76 \pm 0.83$ \\
\hline \multirow{2}{*}{$\mathrm{Na}($ EAM [18]) } & 573 & 11.5 \\
$\mathrm{Na}($ Experiment [24]) & 723 & 16.4 \\
\hline \multirow{2}{*}{$\mathrm{Ce}$ in Na } & 573 & 13 \\
& 723 & $12.30 \pm 0.62$ \\
\multirow{2}{*}{$\mathrm{Pr}$ in $\mathrm{Na}$} & 1000 & $22.92 \pm 0.72$ \\
\hline \multirow{2}{*}{$\mathrm{Nd}$ in $\mathrm{Na}$} & 723 & $8.07 \pm 0.21$ \\
& 923 & $13.73 \pm 0.48$ \\
\hline & 723 & $8.25 \pm 0.29$ \\
& 923 & $12.77 \pm 0.58$ \\
\hline
\end{tabular}

\section{Conclusion:}

In this study, $\mathrm{Ab}$-initio molecular dynamics was employed to investigate the properties of three binary metallic systems with $\mathrm{Ln}$ atoms in liquid $\mathrm{Na}$ at different temperatures. The total pair correlation functions for $\mathrm{Na}-\mathrm{Ce}$ indicate an amorphous state while $\mathrm{Na}-\mathrm{Nd}$ and $\mathrm{Na}-\mathrm{Pr}$ appear to be in the liquid state at typical reactor operation temperatures. The total coordination number was found to decrease with increasing temperature and to increase with the Ln atomic number. Furthermore, it was observed that Ce diffusivity in $\mathrm{Na}$ showed the strongest dependence on temperature when compared with $\operatorname{Pr}$ and Nd. Finally, it was found that dilute concentrations of Pr and Nd had a significant effect on the diffusivity of Na but Ce did not. This may be explained by the segregation phenomena observed in the $\mathrm{Na}-\mathrm{Ce}$ system.

\section{Acknowledgements:}


We acknowledge support from the US Department of Energy, Office of Nuclear Energy through the Nuclear Energy University Program (Project 14-6482) and also acknowledge the computing resources from Idaho National Laboratory.

1 C.W. Arnold, et al. Improvements to Coding of Lanthanide behaviors in Metal Fuel, ANS Winter Meeting 2015, At Washington D.C., Volume: ANS TRANSACTIONS.

2 G.W. Egeland, et al., Reduction of FCCI effects in lanthanide-iron diffusion couples by doping with palladium, J. Nucl. Mater, 440 (2013), 178-192

3 Assel Aitkaliyeva, et al., Fuel Cladding Interaction between U-Pu-Zr Fuel and Fe, Metallurgical and Materials Transactions E, Volume 2, issue 4, pp 220-228, December 2015.

4 R.D. Mariani, et al., Lanthanides in metallic nuclear fuels: Their behavior and methods for their control, J. Nucl. Mater., 419 (2011), 263-271

5 J. Zhang, P. Hosemann, S. Maloy, Models of liquid metal corrosion, Journal of Nuclear Materials 404 (2010) 82-96.

6 M.L. Kuntz, S.F. Corbin, Y. Zhou, Quantifying metallurgical interactions in solid/liquid diffusion couples using differential scanning calorimetry, Acta Materialia 53 (2005) 3071-3082

7 R. Heringer, Ch.-A. Gandin, G. Lesoult, H. Henein, Atomized droplet solidification as an equiaxed growth model, Acta Materialia 54 (2006) 4427-4440

8 T. Yang, et al., Determination of solubility of uranium in liquid sodium, Nuclear Science and Techniques, 19 (2): p. 93-98, April 2008.

9 G. Kresse and J. Furthmüller, Efficiency of ab-initio total energy calculations for metals and semiconductors using a plane-wave basis set. Computational Materials Science, 1996. 6(1): p. 15-50.

10 G. Kresse and J. Furthmüller, Efficient iterative schemes for ab initio total-energy calculations using a plane-wave basis set. Physical Review B, 1996. 54 (16): p. 11169-11186.

11 J. P. Perdew, K. Burke, and M. Ernzerhof, Generalized Gradient Approximation Made Simple. Physical Review Letters, 1996. 77(18): p. 3865-3868. 
12 M. Methfessel and A. T. Paxton, High-precision sampling for Brillouin-zone integration in metals. Physical Review B, 1989. 40(6): p. 3616-3621.

13 Samin, A., Li, X., Zhang, J., Mariani, R. D., and Unal, C., Ab initio molecular dynamics study of the properties of cerium in liquid sodium at $1000 \mathrm{~K}$ temperature. Journal of Applied Physics, 2015. 118(23): p. 234902.

14 Fujio Kakinuma, et al., A structural study of liquid Te-rich Si-Te alloys, Journal of Physics: Conference Series 502 (2014) 012029.

15 Wanqi Jie, Solute redistribution and segregation in solidification processes, Science and Technology of Advanced Materials 2(2001) 29-35

16 E.C. Caldwell, et al., Segregation of elements in high refractory content single crystal nickel based superalloys, TMS(The Minerals, Metals \& Materials Society), 2004

17 A.A. Yuryev, B.R. Gelchinski, Ab initio molecular dynamics study of liquid sodium and cesium up to critical point, AIP Conf. Proc 1673, 020009 (2015)

18 D. K. Belashchenko, Application of the embedded atom model to liquid metals: Liquid sodium. High Temperature, 2009. 47(4): p. 494-507

19 D.B. Miracle, et al., Structure-forming principles for amorphous metals, Materials Science and Engineering A 375-377 (2004) 150-156

20 R. Zeller and P.H. Dederichs, Calculations for the electronic structure of isolated cerium impurities, Physica 130B (1985) 34-36.

21 A.Einstein, On the theory of the brownian movement, Ann.d.Phys. 19(1906) 371-381.

22 S. Vandarkuzhali, P. Venkatesh, Suddhasattwa Ghosh, G. Seenivasan, B. Prabhakara Reddy, T.

Subramanian, N. Sivaraman, and K. Nagarajan, Electrochemistry of rare earth oxy ions REO+ (RE: Ce, $\mathrm{La}, \mathrm{Nd}$ ) in molten $\mathrm{MgCl} 2-\mathrm{NaCl}-\mathrm{KCl}$ eutectic. Journal of Electroanalytical Chemistry, 2007. 611(1-2): p. 181-191. 
23 Jean-François Wax et al., Molecular dynamics simulation study of the interdiffusion properties of liquid Na-K alloys,Journal of Physics: Conference Series 98 (2008) 042001

24 Meyer, R.E. and Nachtrieb, N.H., J. Chem. Phys., 1955, vol. 23, p. 1851. 